\title{
The Ability of Streptomyces spp. Isolated from Iranian Soil to Solubilize Rock Phosphate
}

\author{
Nazila Biglari ${ }^{1 *}$, Hasnuri Mat Hassan ${ }^{1}$, Javid Amini ${ }^{2}$
}

1. School of Biological Sciences, Universiti Sains Malaysia, Penang, Malaysia

2. Department of Microbiology, Academic and Scientific Research Center, Kerman, Iran

\begin{abstract}
Many agricultural soils are deficient in plant available phosphate and hence not able to sustain optimal crop productivity. The problem is due to the ability of many soils to fix phosphate in a wide range of soil $\mathrm{pH}$ and ecological conditions. There is a need to search for more environmental friendly alternatives to improve soil fertility and crop production in phosphate deficient soil. The aim of this study was to isolate, screen, and characterize phosphate solubilizing actinomycetes found in different types of soil with varied $\mathrm{pH}$ from various sites in Iran. Phosphate solubilizing ability of the actinomycetes was evaluated both on modified Pikovskaya's (PVK) agar and into broth media containing Christmas Island Rock Phosphate (CIRP). The abilities of each isolate to solubilize phosphorus was evaluated from day 1 to day 14 after inoculation. Streptomyces spp. were identified morphologically under scanning electron microscope (SEM). About 31\% (22/70) isolates of actinomycetes were found to have the ability to solubilize (CIRP). Isolates IA15 and IA31 showed high solubilizing index (SI) on agar medium whereas isolates IA61, IA59, IA38, IA35, and IA31 were determined to have high CIRP solubilizing ability in broth medium. Isolates IA11, IA31, IA10, and IA61 had high pH decrease in broth medium after 14 days of inoculation. A gradual decrease in $\mathrm{pH}$ was observed over a 14 day period of incubation, suggesting a slow release of phosphate from CIRP. The mechanism of solubilization was related to $\mathrm{pH}$ decrease in broth medium. In general, majority of phosphate solubilizing actinomycetes revealed superior ability to solubilize CIRP.
\end{abstract}

Key words: rock phosphate; solubilizing phosphate; Streptomyces spp;

\section{Introduction}

Among phosphate solubilizing microorganisms (PSM), Actinomycetes are of prime interest since these filamentous sporulating bacteria are able to develop in diverse form of soils (Salcedo et al., 2014). However, they produce various substances

including (anti-fungi, insecticides, anthelminthics, and phytohormone-like compounds that could be useful for plant growth (Castanheira et al., 2014). Among actinobacteria class, the Streptomyces genus leads the great value group of branching soil bacteria due to secondary metabolites

\section{Corresponding author:}

Nazila Biglari

School of Biological Sciences, Universiti Sains Malaysia, Penang, Malaysia

Email: nazila_1979i@yahoo.com

Receive date: 2016-05-17| Accept date: 2016-06-29| Publish date: 2016-07-28

DOI: 10.7575/aiac.abcmed.16.04.03.03

\section{Al}


production, such as antibiotics and extracellular enzymes (Sharma et al., 2014). Streptomyces spp. are an economic producer for vitamins, enzymes, and antibiotics in both environment and soil as the major communities of microbes (Ban et al., 2014). Streptomyces spp. are able to distribute earthy smell and rain adores in environment due to microbial products called geosmins especially after rain (Tsao et al., 2014). Permanent and branched mycelium was seen in Streptomyces spp.

After nitrogen $(\mathrm{N})$, phosphate $(\mathrm{P})$ readily is a major nutrition element limiting plant growth in world-wide agricultural soils especially for crop production (Brännvall et al., 2014). Unfortunately, one of the least present and moving mineral nutrients for plants in the soil is $P$ (Nesme et al., 2014). Therefore, about 0.1\% of the total $P$ is available to plants. Thus, $P$ availability to plant is subjected to chemical fixation in soil with some metal cations which depend on soil pH (Viani et al., 2014). The largest deposit of phosphorus are in rocks and this substance should afford an interesting supply of phosphate fertilizer for yield production (Rodríguez and Fraga, 1999). De Oliveira Mendes et al. (2014) mentioned that in the fields, rock phosphate (RP) is the main phosphate fertilizers. They also suggested that natural RP has been found as a source of $P$ in a wide area with different soil types specially acid soil which is a valuable alternative and less expensive natural source for phosphate fertilizer. The weakly soluble RP is used in agriculture as a natural slow releasing phosphate fertilizer (Rahman et al., 2014). The favorable effect of rock phosphate has made this material an attractive element for application in agriculture (Barea et al., 2002).

In this research study, we studied on $P$ solubilizing ability of isolated Streptomyces spp. The significance of this finding may lead to a novel actinomycetes solubilizing rock phosphate that has the potential to be utilized to increase soil fertility and crop productivity.

\section{Materials and Methods}

\subsection{Screening of Streptomyces spp.}

The strains isolated from soil sources according to $\mathrm{P}$ solubilization abilities were coded in IA1 to IA70. For screening Streptomyces spp. the Malt Extract Agar (MEA) medium at $280 \mathrm{C}$ temperature for 3 days incubation was used containing (per liter): maltose $12.75 \mathrm{~g}$, dextrin $2.75 \mathrm{~g}$, glycerol $2.35 \mathrm{~g}$, peptone $0.78 \mathrm{~g}$, and agar $15 \mathrm{~g}$ (Busarakam et al., 2014). Then, gram stain from cover slip culturing and acid-fast stain were used to identify the genus (Staneck and Roberts, 1974).

2.2 Qualification of Total Free Inorganic Phosphate

For study on $\mathrm{P}$ abilities, PVK agar medium was used at $28^{\circ} \mathrm{C}$ temperature for 14 days (Mehta et al., 2014). The halo zone due to dissolving phosphate into inorganic phosphate which can used by microorganism was measured at 1st, 7th, and 14th day of incubation. All experiments were replicated in three times. The index of solubility was calculated using the formula:

Solubilization index $(\mathrm{SI})=$ Colony diameter + Halo zone diameter/ Colony diameter (Khan et al., 2014).

2.3 Quantitation Total Free Inorganic Phosphate

Molybdenum blue technique was chosen for determination of available P (Ngosong et al., 2014).The fresh cultures of strains were inoculated into the modified Pikovskaya's (PVK) broth with RP (30\%), followed by shaking at 180 rpm and a temperature range of $28-30^{\circ} \mathrm{C}$ for 14 days. $10 \mathrm{ml}$ of suspension of inoculate was taken out and centrifuged at $1500 \mathrm{rpm}$ for 15 min (sigmacentrifuge, Model 4K15).The supernatant was reacted with the reagents to obtain blue colour. Concentration 
determination of soluble inorganic phosphate pi was determined spectroscopically at $880 \mathrm{~nm}$ wavelength. All treatments were replicated in three times.

2.4 Measurement of $\mathrm{pH}$ During Phosphate Solubilizing Activity by Selected Isolates

The $\mathrm{pH}$ changes in the pi solubilizing process was measured to find the relationship between dissolved $\mathrm{Pi}$ and $\mathrm{pH}$ values. After taking out 5 $\mathrm{ml}$ of supernatant to react with reagents, the rest of aliquot were used to measure $\mathrm{pH}$ values with a pH meter (Orion, USA). The measurement of $\mathrm{pH}$ was concurrently performed with $\mathrm{P}$ determination of each aliquot (Salcedo et al., 2014).

\subsection{Statistical Analysis}

The results and data were evaluated by oneway analysis of variance (ANOVA) and significance of differences between means and SE were tested using Duncan's Multiple Range Test at $p<0.05$ by SPSS Statistics 20 IBM $^{\circledR}$.

\section{Results}

3.1 Isolation of Phosphate Solubilizing Actinomycetes

Actinomycetes were identified morphologically by microscopic observation based on the characteristics of spores (spore formation on the substrate and/or aerial mycelium and presence of single spores or sporangia), hyphae, pigmentation, and mycelia fragmentation.

The morphological characteristics of 22 isolates based on elevation, edge, pigmentation, and surface appearances were recorded. From 22 isolates, 14 were irregular, 5 were filamentous, 2 were rhizoid, and 1 isolates was circular. 15 strains of actinomycetes were dry, rough, and penetrating agar media. In some cases, colonies were sticky, smooth, and glistening. Different pigmentation, edge, and elevation of colonies were observed among all isolates as well (Table 1).
3.2 Inorganic Phosphate (Pi) Release by Phosphate Solubilizing Streptomyces Strains

Qualification of $\mathrm{Pi}$ release: Isolates IA61 showed high Pi released on modified PKV agar medium while isolates IA22, IA15, and IA31 were significantly lower $\mathrm{Pi}$ release on 1st day of inoculation (Table 2). Whereas at 7th days after inoculation, IA22 showed the highest $\mathrm{Pi}$ released followed by IA15, IA61, and IA27 on modified PKV agar medium. Finally, at 14th day of inoculation, isolates IA15 and IA31 had the highest $\mathrm{Pi}$ released on modified $\mathrm{PKV}$ agar medium.

Quantitation of $\mathrm{Pi}$ release: At 1st day of incubation, IA61 released significantly highest Pi release. During 7 days of incubation, IA11 released more $\mathrm{Pi}$ and followed by strains IA31, IA8, IA38, and IA59. The highest Pi released was determined in isolate IA61 after 14 days of incubation at $28^{\circ} \mathrm{C}$. Similarly, isolates IA59, IA38, IA35, and IA31 were also dissolved high amount of $\mathrm{Pi}$ at the same period of incubation at $28^{\circ} \mathrm{C}$.

3.3 Relationship between Solubilized $\mathrm{Pi}$ Concentration and $\mathrm{pH}$

A decrease in $\mathrm{pH}$ of the media was observed with all isolates of Actinomycetes (Table 4). It was found that isolates IA61, IA8, IA22, IA31, $I A 27, I A 13, I A 10, I A 12$, and IA59 had a high $\mathrm{pH}$ decrease in modified PVK broth medium after 1 day of incubation. Isolate IA10 had the highest $\mathrm{pH}$ decrease in modified PVK broth medium after 7 days of incubation. After 14 days of incubation, $\mathrm{pH}$ of isolates IA11, IA31, IA10, and IA61 was decreased in modified PVK broth medium. Generally, $\mathrm{pH}$ of isolates IA12, and IA13 was increased during incubation period and $\mathrm{pH}$ of isolate IA27 was increased from 1st day to 7 th day of incubation and then $\mathrm{pH}$ was decreased at 14th days of incubation. 


\begin{tabular}{|c|c|c|c|c|c|c|c|}
\hline $\begin{array}{l}\text { Isolated } \\
\text { colony }\end{array}$ & $\begin{array}{c}\text { Form of whole } \\
\text { colony }\end{array}$ & Elevation & Edge & Pigmentation & Surface & $\begin{array}{l}\text { Gram } \\
\text { stain }\end{array}$ & $\begin{array}{c}\text { Species } \\
\text { identification }\end{array}$ \\
\hline IA6 & Filamentous & Raised & Filamentous & $\begin{array}{l}\text { White-light } \\
\text { pink }\end{array}$ & $\begin{array}{l}\text { Powdery, dry, rough, } \\
\text { penetrating agar media }\end{array}$ & + & Streptomyces sp. \\
\hline IA7 & Irregular & Raised & Undulate & White & $\begin{array}{l}\text { Powdery, dry, rough, } \\
\text { penetrating agar media }\end{array}$ & + & Streptomyces sp. \\
\hline IA8 & Filamentous & Raised & Filamentous & $\begin{array}{l}\text { White-light } \\
\text { pink }\end{array}$ & $\begin{array}{l}\text { Powdery, dry, rough, } \\
\text { penetrating agar media }\end{array}$ & + & Streptomyces sp. \\
\hline IA10 & Rhizoid & Flat & Undulate & Pale-yellow & Sticky, smooth, glistening & + & Streptomyces sp. \\
\hline IA11 & Circular & Umbonate & & Orange & Smooth, glistening & + & Streptomyces sp. \\
\hline IA12 & Irregular & $\begin{array}{c}\text { Convex or } \\
\text { dome }\end{array}$ & Undulate & Yellow & Smooth, glistening & + & Streptomyces sp. \\
\hline IA13 & Filamentous & Raised & Filamentous & $\begin{array}{l}\text { White-light } \\
\text { pink }\end{array}$ & $\begin{array}{l}\text { Powdery, dry, rough, } \\
\text { penetrating agar media }\end{array}$ & + & Streptomyces sp. \\
\hline IA14 & Irregular & $\begin{array}{c}\text { Convex or } \\
\text { dome }\end{array}$ & Entire & Yellow & Sticky & + & Streptomyces sp. \\
\hline IA15 & Irregular & Flat & Entire & Pale-yellow & Smooth, glistening & + & Streptomyces sp. \\
\hline IA17 & Irregular & Raised & Undulate & Brown-black & $\begin{array}{c}\text { Dry, rough, penetrating } \\
\text { agar media }\end{array}$ & + & Streptomyces sp. \\
\hline IA18 & Rhizoid & Flat & Undulate & Pale-yellow & Smooth, glistening & + & Streptomyces sp. \\
\hline IA19 & Filamentous & Raised & Filamentous & $\begin{array}{l}\text { White-light } \\
\text { pink }\end{array}$ & $\begin{array}{l}\text { Powdery, dry, rough, } \\
\text { penetrating agar media }\end{array}$ & + & Streptomyces sp. \\
\hline IA22 & Irregular & Raised & Undulate & White-red & $\begin{array}{l}\text { Dry, rough, penetrating } \\
\text { agar media }\end{array}$ & + & Streptomyces sp. \\
\hline IA24 & Irregular & Raised & Undulate & White & $\begin{array}{l}\text { Powdery, dry, rough, } \\
\text { penetrating agar media }\end{array}$ & + & Streptomyces sp. \\
\hline IA27 & Irregular & Umbonate & Curled & Gray-white & $\begin{array}{l}\text { Powdery, dry, rough, } \\
\text { penetrating agar media }\end{array}$ & + & Streptomyces sp. \\
\hline IA28 & Irregular & Raised & Undulate & White & $\begin{array}{l}\text { Powdery, dry, rough, } \\
\text { penetrating agar media }\end{array}$ & + & Streptomyces sp. \\
\hline IA31 & Irregular & Umbonate & Curled & Gray-brown & $\begin{array}{l}\text { Dry, rough, } \\
\text { penetrating }\end{array}$ & + & Streptomyces sp. \\
\hline IA35 & Filamentous & Raised & Filamentous & $\begin{array}{l}\text { White-light } \\
\text { pink }\end{array}$ & $\begin{array}{l}\text { Powdery, dry, rough, } \\
\text { penetrating agar media }\end{array}$ & + & Streptomyces sp. \\
\hline IA38 & Irregular & Raised & Entire & White & $\begin{array}{l}\text { Powdery, dry, rough, } \\
\text { penetrating agar media }\end{array}$ & + & Streptomyces sp. \\
\hline IA56 & Irregular & Raised & Entire & White-yellow & $\begin{array}{l}\text { Powdery, dry, rough, } \\
\text { penetrating agar media }\end{array}$ & + & Streptomyces sp. \\
\hline IA59 & Rhizoid & Flat & Undulate & Pale-yellow & Smooth, glistening & + & Streptomyces sp. \\
\hline IA60 & Irregular & $\begin{array}{c}\text { Convex or } \\
\text { dome }\end{array}$ & Undulate & Yellow & Smooth, glistening & + & Streptomyces sp. \\
\hline IA61 & Irregular & Raised & Undulate & White & $\begin{array}{l}\text { Dry, rough, powdery, } \\
\text { penetrating agar media }\end{array}$ & + & Streptomyces sp. \\
\hline
\end{tabular}

Table 1: The colony characteristics based on substrate mycelium colour and colony morphology of 22 different actinomycetes colonies isolated on MEA agar plates for 3 days. 

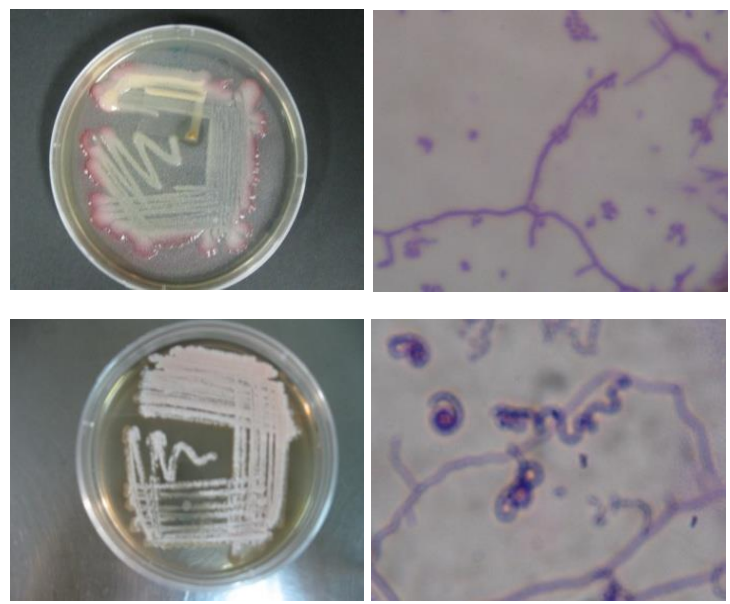

Figure 1: Colony Morphology Observation

Description: Streptomyces. sp with glistening colony on MEA medium (top, left side)

Streptomyces. sp. with aerial mycelium with acid fast staining in purple colour (top, right side,

$10 \mu \mathrm{m}$ ) Streptomyces. sp with powdery/ dry/ white-pink colony on MEA medium (bottom, left side) Streptomyces. sp. with spiral mycelium with acid fast staining in purple colour (bottom, right side, $10 \mu \mathrm{m}$ ). After 3 days of inoculation at $28^{\circ} \mathrm{C}$ (Table 3 ).

\begin{tabular}{|c|c|c|c|}
\hline \multirow{2}{*}{ Actinomycete } & \multicolumn{3}{|c|}{ (Mean \pm SE) Solubilizing index (SI) } \\
\hline & Day 1 & Day 7 & Day 14 \\
\hline IA6 & $2.07 \pm 0.03^{c}$ & $2.12 \pm 0.08^{\mathrm{de}}$ & $2.25 \pm 0.05^{\text {ef }}$ \\
\hline IA7 & $2.11 \pm 0.01^{c}$ & $2.10 \pm 0.00 \mathrm{de}$ & $2.10 \pm 0.00^{f}$ \\
\hline IA8 & $2.03 \pm 0.02^{c}$ & $2.03 \pm 0.00^{e}$ & $2.09 \pm 0.07^{f}$ \\
\hline IA10 & $2.15 \pm 0.05^{c}$ & $1.94 \pm 0.09^{e}$ & $2.19 \pm 0.08^{e f}$ \\
\hline IA11 & $2.20 \pm 0.00^{b c}$ & $2.30 \pm 0.01 \mathrm{de}$ & $2.22 \pm 0.08$ ef $^{-1}$ \\
\hline IA12 & $2.05 \pm 0.01^{c}$ & $2.29 \pm 0.09 \mathrm{de}$ & $2.20 \pm 0.00$ ef \\
\hline IA13 & $2.13 \pm 0.01^{c}$ & $2.12 \pm 0.06^{\mathrm{de}}$ & $2.06 \pm 0.00^{f}$ \\
\hline IA14 & $2.14 \pm 0.05^{c}$ & $2.09 \pm 0.01 \mathrm{de}$ & $2.14 \pm 0.06$ ef $^{-14}$ \\
\hline IA15 & $3.18 \pm 0.02 b c$ & $3.98 \pm 0.22^{a}$ & $6.01 \pm 0.37^{\mathrm{a}}$ \\
\hline IA17 & $2.10 \pm 0.00^{c}$ & $2.10 \pm 0.06^{\mathrm{de}}$ & $2.10 \pm 0.00^{f}$ \\
\hline IA18 & $3.15 \pm 0.35^{b c}$ & $2.97 \pm 0.09^{c}$ & $2.99 \pm 0.06^{b c}$ \\
\hline IA19 & $2.03 \pm 0.26^{c}$ & $2.27 \pm 0.01 \mathrm{de}$ & $2.20 \pm 0.07^{\text {ef }}$ \\
\hline IA22 & $3.30 \pm 0.15^{b c}$ & $4.93 \pm 0.26^{b c}$ & $3.26 \pm 0.20^{b}$ \\
\hline IA24 & $2.24 \pm 0.13^{b c}$ & $2.18 \pm 0.07^{d e}$ & $2.18 \pm 0.07^{e f}$ \\
\hline IA27 & $2.92 \pm 0.28^{b}$ & $3.13 \pm 0.07^{c}$ & $3.02 \pm 0.19 b c$ \\
\hline IA28 & $2.21 \pm 0.00^{b c}$ & $2.24 \pm 0.04 \mathrm{de}$ & $2.21 \pm 0.01 b c$ \\
\hline IA31 & $3.17 \pm 0.11^{a}$ & $2.92 \pm 0.35^{c}$ & $4.06 \pm 0.26^{e f}$ \\
\hline IA35 & $2.20 \pm 0.06^{b c}$ & $2.14 \pm 0.00^{d e}$ & $2.14 \pm 0.00^{e f}$ \\
\hline IA38 & $2.11 \pm 0.00^{c}$ & $2.13 \pm 0.03^{d e}$ & $2.11 \pm 0.00^{f}$ \\
\hline IA56 & $2.05 \pm 0.05^{c}$ & $2.12 \pm 0.06^{\mathrm{de}}$ & $2.08 \pm 0.01^{f}$ \\
\hline IA59 & $2.06 \pm 0.06^{c}$ & $2.03 \pm 0.01 \mathrm{de}$ & $2.04 \pm 0.02^{f}$ \\
\hline IA60 & $2.06 \pm 0.02^{\mathrm{c}}$ & $2.07 \pm 0.03^{d e}$ & $2.11 \pm 0.07^{e f}$ \\
\hline IA61 & $4.18 \pm 0.25^{a}$ & $3.21 \pm 0.17^{b c}$ & $3.18 \pm 0.19^{b}$ \\
\hline
\end{tabular}

Table 2: Solubilizing index (SI) of 22 solubilizing actinomycetes on D1, D7, and D14 of inoculation at $28^{\circ} \mathrm{C}$. 
*Mean values in the same column indicated by different superscript letters are significantly different (Duncan test, $\mathrm{p}<$ 0.05).

\begin{tabular}{|c|c|c|c|}
\hline \multirow{2}{*}{ Actinomycete } & \multicolumn{3}{|c|}{$($ Mean $\pm S E)$ Pi release $(\mu \mathrm{g} / \mathrm{I})$} \\
\hline & Day 1 & Day 7 & Day 14 \\
\hline IA6 & $3.1 \pm 0.04$ defg & $4.1 \pm 0.13^{b c d e}$ & $4.9 \pm 0.05^{b c d e f}$ \\
\hline IA7 & $4.3 \pm 0.04^{b c d e f g}$ & $4.7 \pm 0.15^{b c d e}$ & $4.6 \pm 0.14^{\mathrm{cdefg}}$ \\
\hline IA8 & $5.5 \pm 0.16^{b c d}$ & $5.4 \pm 0.11$ a bce & $3.7 \pm 0.02$ defgh \\
\hline IA10 & $5.8 \pm 0.07^{b c d}$ & $4.1 \pm 0.15^{b c d e}$ & $3.5 \pm 0.10 \mathrm{defgh}$ \\
\hline IA11 & $4.2 \pm 0.01^{b c d e f g}$ & $6.0 \pm 0.01$ a be & $3.9 \pm 0.03$ defgh \\
\hline IA12 & $3.9 \pm 0.14^{b c d e f g}$ & $3.6 \pm 0.01 b c d e$ & $2.2 \pm 0.04^{\mathrm{gh}}$ \\
\hline IA13 & $5.5 \pm 0.17^{b c d}$ & $2.9 \pm 0.00^{b c d e}$ & $2.8 \pm 0.02$ efgh \\
\hline IA14 & $5.2 \pm 0.11^{b c d e}$ & $4.1 \pm 0.16^{b c d e}$ & $3.1 \pm 0.03$ efgh \\
\hline IA15 & $3.7 \pm 0.01 \mathrm{bcde}$ & $4.5 \pm 0.13^{b c d e}$ & $4.3 \pm 0.05^{\mathrm{cdefg}}$ \\
\hline IA17 & $2.4 \pm 0.02$ efg & $4.9 \pm 0.02^{b c d e}$ & $2.3 \pm 0.07^{\mathrm{fgh}}$ \\
\hline IA18 & $2.9 \pm 0.03^{d c f g}$ & $4.2 \pm 0.05^{b c d c}$ & $3.6 \pm 0.02 \mathrm{dcfgh}$ \\
\hline IA19 & $3.1 \pm 0.09$ de $f g$ & $3.6 \pm 0.02^{b c d e}$ & $3.5 \pm 0.04$ defgh \\
\hline IA22 & $5.3 \pm 0.03^{b c d e}$ & $2.5 \pm 0.12^{\mathrm{cde}}$ & $3.1 \pm 0.01$ efgh \\
\hline IA24 & $1.9 \pm 0.04^{\mathrm{fg}}$ & $2.9 \pm 0.03^{b c d e}$ & $3.0 \pm 0.02$ efgh \\
\hline IA27 & $4.9 \pm 0.07^{b c d e f}$ & $2.5 \pm 0.03^{\mathrm{cde}}$ & $4.3 \pm 0.03^{\mathrm{cdefgh}}$ \\
\hline IA28 & $4.6 \pm 0.08^{b c d e f g}$ & $3.8 \pm 0.04 b c d e$ & $4.8 \pm 0.05$ bcdefg \\
\hline IA31 & $6.2 \pm 0.04^{b c}$ & $5.5 \pm 0.13^{a b c e}$ & $5.0 \pm 0.06^{b c d e}$ \\
\hline IA35 & $4.5 \pm 0.02^{b c d e f g}$ & $3.7 \pm 0.03^{b c d e}$ & $5.1 \pm 0.04^{b c d e}$ \\
\hline IA38 & $4.4 \pm 0.08^{b c d e f g}$ & $5.0 \pm 0.12^{b c d e}$ & $5.4 \pm 0.04^{b c d e}$ \\
\hline IA56 & $3.9 \pm 0.05^{b c d e f g}$ & $4.3 \pm 0.06^{b c d e}$ & $3.6 \pm 0.03$ defgh \\
\hline IA59 & $6.9 \pm 0.08^{a b}$ & $5.0 \pm 0.17^{b c d e}$ & $5.9 \pm 0.10^{b c d}$ \\
\hline IA60 & $6.9 \pm 0.18^{a b}$ & $3.3 \pm 0.04$ bcde & $4.6 \pm 0.18^{\mathrm{cdefg}}$ \\
\hline IA61 & $9.0 \pm 0.13^{a}$ & $4.0 \pm 0.00^{b c d e}$ & $7.2 \pm 0.20^{a b}$ \\
\hline
\end{tabular}

Table 3: Inorganic P Release $(\mu \mathrm{g} / \mathrm{l})$ by Phosphate Solubilizing Actinomycetes.

*Mean values in the same column indicated by different superscript letters are significantly different (Duncan test, $p<$ 0.05).

\begin{tabular}{|c|c|c|c|c|}
\hline \multirow{2}{*}{ Actinomycete } & \multicolumn{4}{|c|}{ (Mean $\pm \mathrm{SE}$ ) pH decrease } \\
\hline & \multicolumn{2}{|c|}{ Day 1} & \multirow{2}{*}{$\frac{\text { Day } 7}{5.01 \pm 0.07^{h}}$} & \multirow{2}{*}{$\frac{\text { Day } 14}{4.14 \pm 0.03^{b c d e}}$} \\
\hline & IA6 & $5.36 \pm 0.08^{\text {cdefgh }}$ & & \\
\hline & IA7 & $5.63 \pm 0.32^{\text {efgh }}$ & $4.72 \pm 0.07$ defgh & $4.36 \pm 0.05 b c d e$ \\
\hline & IA8 & $4.56 \pm 0.22 \mathrm{ab}$ & $4.14 \pm 0.12$ abcd & $4.07 \pm 0.13^{b c d}$ \\
\hline & IA10 & $4.88 \pm 0.25^{\mathrm{ab}}$ & $3.95 \pm 0.03^{\mathrm{ab}}$ & $3.92 \pm 0.04 b c$ \\
\hline & IA11 & $5.82 \pm 0.29 \mathrm{gh}$ & $4.29 \pm 0.12^{\text {abcdefg }}$ & $3.63 \pm 0.37^{a b}$ \\
\hline & IA12 & $4.91 \pm 0.11 \mathrm{abcde}$ & $6.50 \pm 0.20^{j}$ & $6.49 \pm 0.03^{f}$ \\
\hline & IA13 & $4.87 \pm 0.05 a b c d$ & $5.80 \pm 0.31^{i}$ & $6.10 \pm 0.11^{f}$ \\
\hline & IA14 & $5.55 \pm 0.24$ defgh & $4.21 \pm 0.26$ abcde & $4.22 \pm 0.27^{b c d e}$ \\
\hline & IA15 & $5.32 \pm 0.05^{\mathrm{cdefgh}}$ & $4.83 \pm 0.10^{\mathrm{fgh}}$ & $4.18 \pm 0.24 \mathrm{bcde}$ \\
\hline & IA17 & $5.93 \pm 0.47^{h}$ & $4.39 \pm 0.15^{b c d e f g}$ & $4.35 \pm 0.43^{b c d e}$ \\
\hline & IA19 & $5.67 \pm 0.05^{f g h}$ & $4.25 \pm 0.03$ abcdefg & $4.82 \pm 0.12^{\mathrm{cde}}$ \\
\hline & IA22 & $4.76 \pm 0.39 \mathrm{abc}$ & $4.38 \pm 0.13^{b c d e f g}$ & $4.39 \pm 0.20 \mathrm{bcde}$ \\
\hline & IA24 & $6.59 \pm 0.29^{i}$ & $4.38 \pm 0.03^{b c d e f g}$ & $5.02 \pm 0.04^{\mathrm{e}}$ \\
\hline & IA27 & $4.86 \pm 0.07^{a b c d}$ & $5.02 \pm 0.54^{h}$ & $4.91 \pm 0.99 \mathrm{de}$ \\
\hline & IA28 & $5.22 \pm 0.06^{\text {bcdefgh }}$ & $4.72 \pm 0.04$ defgh & $4.27 \pm 0.14 \mathrm{bcde}$ \\
\hline & IA31 & $4.80 \pm 0.19^{h i j}$ & $4.42 \pm 0.05^{b c d e f g}$ & $3.72 \pm 0.21^{\mathrm{ab}}$ \\
\hline & IA35 & $5.61 \pm 0.09$ efgh & $4.49 \pm 0.12^{b c d e f g h}$ & $4.10 \pm 0.10^{b c d e}$ \\
\hline
\end{tabular}




$$
\begin{array}{lll}
\text { IA38 } 5.66 \pm 0.17^{\mathrm{fgh}} & 4.80 \pm 0.15^{\mathrm{efgh}} & 4.01 \pm 0.07^{\mathrm{bcd}} \\
\text { IA56 } 5.47 \pm 0.28^{\mathrm{cdefgh}} & 4.40 \pm 0.05^{\mathrm{bcdefg}} & 4.41 \pm 0.10^{\mathrm{bcde}}
\end{array}
$$

\begin{tabular}{lll}
\hline \multicolumn{2}{c}{ (Mean \pm SE) pH decrease } & \\
Day 1 & Day 7 & Day 14 \\
\hline IA60 $5.21 \pm 0.14^{\text {bcdefgh }}$ & $4.60 \pm 0.03^{\text {cd efg h }}$ & $4.34 \pm 0.14^{\text {bcd e }}$ \\
IA61 $4.49 \pm 0.20^{\text {a }}$ & $4.10 \pm 0.01^{\text {abc }}$ & $3.93 \pm 0.09^{\text {bc }}$ \\
\hline
\end{tabular}

Table 4: $\mathrm{pH}$ Decrease of Media Containing Phosphate Solubilizing Actinomycetes with CIRP

*Mean values in the same column indicated by different superscript letters are significantly different (Duncan test, $p<$ 0.05).
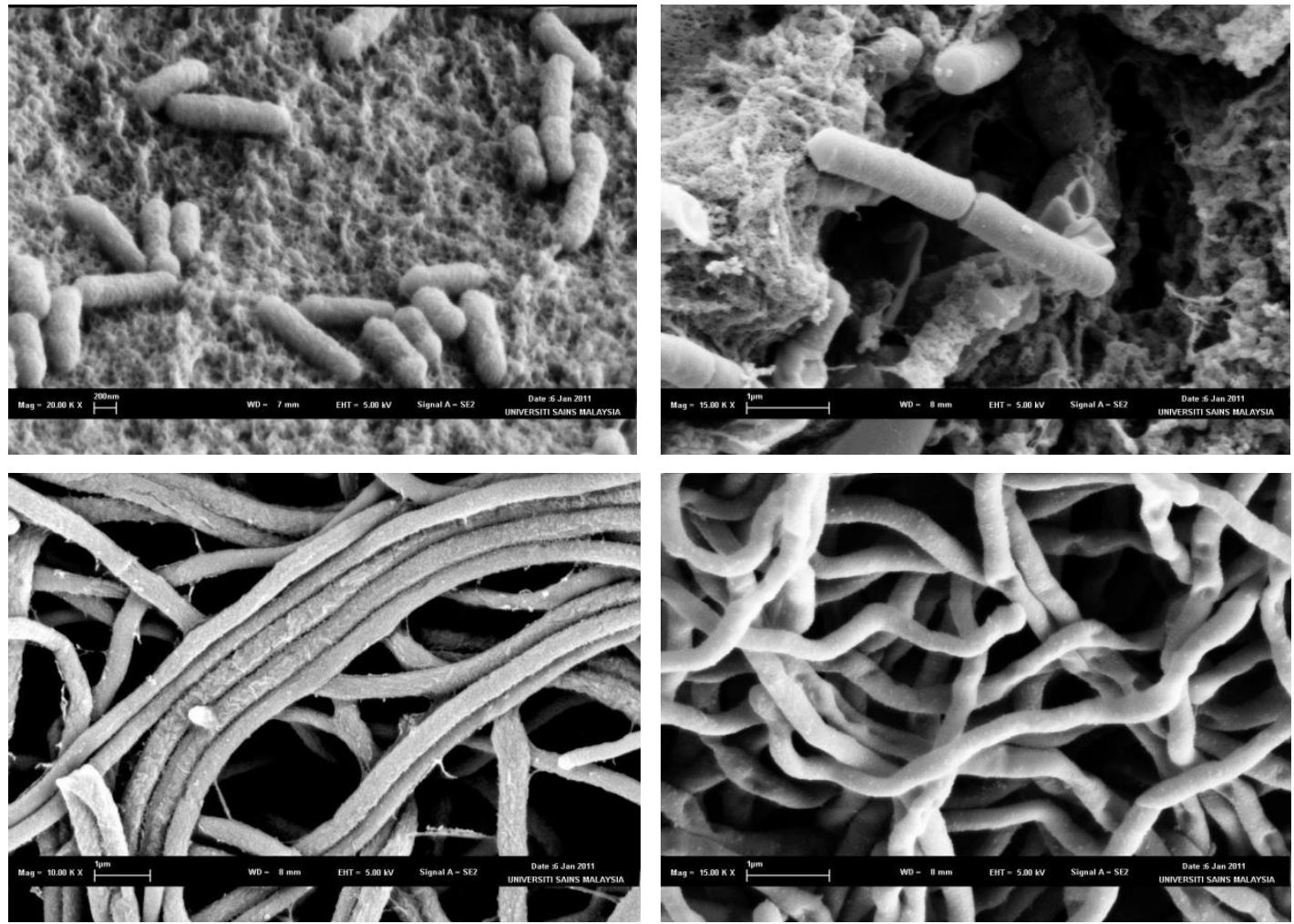

Plate 2: Scanning Electron Microscope (SEM) Observation

Description: Colony morphology of actinomycetes isolates under scanning electron microscope (SEM) (top, left side) isolate IA59, bacilli form, Streptomyces sp. (top, right side) isolate IA10, bacilli form, Streptomyces sp. (bottom, left side) isolate IA8, extensively branching filament, Streptomyces sp. (bottom, right side) isolate IA35, extensively branching filament, Streptomyces sp.

3.4 Morphology Observation under Scanning Electron Microscope (SEM)

Vegetative cell, extensively branching filament and bacilli form of 8 Streptomyces spp. (IA8, IA10, IA35, and IA59) were observed under scanning electron microscope (SEM) in Plate 2.

\section{Discussion}

4.1 Phosphate Solubilizing Index (SI) of Phosphate Solubilizing Actinomycetes

Actinomycetes isolates in the present study showed different ability of phosphate solubilization as shown in the value of SI (Table 2). One third of the isolates were obtained with decrease in SI value with continues incubation until 14th days. The decrease in SI of strains from 1 st to 7 th day of incubation were 
reported due to consumption of released $\mathrm{P}$ by the strains (Hamdali et al., 2008). Gupta et al. (2010) found that five Streptomyces species isolated from the phyllosphere of Heritiera fomes, showed optimum solubilization at 15 days of incubation which gradually declined upon extension to 21 days. This could be the explanation on why SI were decreased after some incubation period.

The lower value of $\mathrm{SI}$ in the current study (1.94 to 6.01) could be due to the type of insoluble phosphate which was used i.e. Christmas Island Rock Phosphate (CIRP) and/ or species of actinomycetes. Previous study was done by Sahu et al. (2007) using Kuster's agar medium supplemented with $\mathrm{CaHPO} 4(5 \mathrm{~g} / \mathrm{l})$ as the sole source of phosphate. The $\mathrm{SI}$ of actinomycetes strains were in the range of $10.82 \pm 0.7$ to $41.66 \pm 2.3$ (Sahu et al., 2007). In Turkey, the SI of Streptomyces spp. inoculated on PVK agar media supplemented with tricalcium phosphate incubated at $28^{\circ} \mathrm{C}$ after 3 days was in the range of 3.6 to 28.5 (Tallapragada and Seshachala, 2012). In another study among 10 phosphate solubilizing isolate, the phosphate solubilization index ranged 12 to 32 (Kaviyarasi et al., 2011).

4.2 $\mathrm{Pi}$ Release $(\mu \mathrm{g} / \mathrm{l})$ of Phosphate Solubilizing Actinomycetes

The present study demonstrated that the ability of PSA to solubilize CIRP varied between isolates of actinomycetes from various sampling sites in Iran, some being more efficient than others. This findings suggest that isolates IA61 had high abilities for enrichment of agriculture soils with inorganic phosphorus. The release of phosphate by phosphate solubilizing actinomycete isolates was $9.00 \mu \mathrm{g} / \mathrm{l}$ as being the most and $1.90 \mu \mathrm{g} / \mathrm{l}$ as being the least. The small release was in accordance with the need of phosphate by the growing actinomycetes in the solution culture. This is one of the important characteristics of a biofertilizer. A bio-fertilizer should have the ability to slowly release nutrients from a source. Other studies also reported varied amount of phosphate solubilized in solution cultures. In earlier study, strains isolated from research in Colombia had phosphorus solubilizing capacities between 396 and $525 \mu \mathrm{g} / \mathrm{ml}$ with tricalcium phosphate (Salcedo et al., 2014).Whereas the 22 isolates were able to solubilize the tri-calcium phosphate ranged from $315.46 \mu \mathrm{g} / \mathrm{mg}$ to $50.14 \mu \mathrm{g} / \mathrm{mg}$ (Sujatha and Ammani, 2014). Another study done in India, selected isolates could dissolved inorganic phosphate of $\mathrm{Ca} 3(\mathrm{PO} 4) 2$ within National Botanical Research Institute Phosphate (NBRIP) liquid medium with highest concentration of $164.1 \pm 4.1 \mu \mathrm{g} / \mathrm{ml}$ and $145.1 \pm 2.7 \mu \mathrm{g} / \mathrm{ml}$ respectively. Although, the lowest concentration in same study was found in Streptomyces sp. with $89.3 \pm 3.1 \mu \mathrm{g} / \mathrm{ml}$ (Dastager and Damare, 2013). While Streptomyces spp. showed $92 \mathrm{mg} / \mathrm{l}$ Pi released from the solubilization of tri-calcium phosphate in culture medium (Sadeghi et al., 2012). In another study, phosphate solubilized in PVK liquid medium ranged between 55.60 and $168.30 \mathrm{mg} / \mathrm{L}$ (Karagöz et al., 2012). Release of Pi by different isolates of actinomycetes was between 0.08 to $0.01 \mathrm{mg} / \mathrm{g}$ in broth medium (Sahu et al., 2007). Hamdali et al. (2012) reported five selected actinomycetes strains showed different abilities to release phosphate i.e. from 4.38 to $25.87 \mu \mathrm{g} / \mathrm{ml}$. In India, the quantitative estimation of phosphate solubilization by Strepmtomeces spp. reported diverse concentration of phosphate solubilizing ability ranging from 4.80 to $26.50 \mathrm{mg} / 100 \mathrm{ml}$ (Gangwar et al., 2012). The maximum level of phosphorus solubilization potential of isolates selected from tropical soils was established about $1727 \mu \mathrm{g} / \mathrm{ml}$ of Ca-P and $48.0 \mu \mathrm{g} / \mathrm{ml}$ of RP. In same experimental work, the other group of isolates obtained $1703 \mu \mathrm{g} / \mathrm{ml}$ solubility of Ca-P and $34.5 \mu \mathrm{g} / \mathrm{ml}$ of RP (Asuming-Brempong and Aferi, 2014). In contrast, fungus (Trichoderma spp.) isolated from rhizosphere of pine, deodar, 
bamboo, guava and oak showed $\mathrm{P}$ solubilizing potential using NBRIP broth containing tricalcium phosphate (TCP) as the sole $\mathrm{P}$ source. They recorded maximum amount of soluble phosphate about $404.07 \mu \mathrm{g} / \mathrm{ml}$ after $96 \mathrm{~h}$ of incubation at $30+1^{\circ} \mathrm{C}$ (Kapri and Tewari, 2010).

The concentration of Pi released in the PVK broth media indicated various efficiency of actinomycete isolates to solubilized CIRP. In general, the release of $\mathrm{Pi}$ concentration was higher in the beginning of incubation period due to $P$ availability. The different source of phosphate and isolates as well as incubation period might be the factors that contribute to the difference in amount of $\mathrm{Pi}$ released. Regarding incubation period, no achievement by time even after 60 days of incubation on $P$ equilibrium (Rajput et al., 2014). However, it was reported that actinomycete isolates showed optimum solubilization at 15 days of incubation which gradually declined upon extension to 21 days (Gupta et al., 2010). On the other hand, the concentration of $\mathrm{Pi}$ in solution culture also decreased or increased over incubation periods depends on enzyme mechanisms (Sahu et al., 2007). While a study by Coutinho, (2012) showed that the maximum concentration of $\mathrm{Pi}$ was obtained seven days after inoculation (Achal et al., 2007, Coutinho et al., 2012).

4.3 pH Decrease of Phosphate Solubilizing Actinomycetes

In this study, isolates that solubilized large amount of $\mathrm{Pi}$ were also showing high solubilizing index (SI) and $\mathrm{pH}$ decrease. The $\mathrm{pH}$ decreased from the initial value of $7.00 \pm 0.02$ to 3.63 within PVK broth culture after 14 days of incubation period. This decrease was more than $3 \mathrm{pH}$ units. In most cases gradual $\mathrm{P}$ solubilization process of up to $14 \mathrm{~d}$, indicated the slow action of actinomycetes on CIRP. Gradual change in $\mathrm{pH}$ observed in all isolates, confirmed this statement. Total phosphorus concentration of $360 \mathrm{mg} / \mathrm{kg}$ with only 118 $\mathrm{mg} / \mathrm{kg}$ of available form obtained with $\mathrm{pH}$ around 5 or less (Salcedo et al., 2014). In a similar study Sahu et al. (2007) showed that $\mathrm{pH}$ change gradually decreased upon incubation. Earlier reports also indicated reduction in $\mathrm{pH}$ during $\mathrm{P}$ solubilization by microbes (Coutinho et al., 2012). Earlier study showed that maximum solubilization of phosphate occurred within 2 weeks period after inoculation under controlled conditions (Ogbo, 2010), which could be due to the release of acidic substances produced by the strain during incubation (Sahu et al., 2007).

The mechanisms of microbial solubilization of phosphate has been proposed as may be due to the excretion of organic acids which was indicated by acidification of the broth medium (Whitelaw, 1999) or to the excretion of chelating substances (such as siderophores) that form stable complexes with phosphorus adsorbents (aluminum, iron and calcium (AlHalbouni et al., 2008). Earlier study showed the decrease in $\mathrm{pH}$ of culture filtrate confirmed the assertion that phosphate solubilizing microorganisms solubilize insoluble phosphates mainly by secreting organic acids into the medium (Gupta et al., 2010).

\section{Conclusion}

Seventy (70) actinomycetes of different morphological characteristics were isolated from various soils and water samples in Iran. About $31 \%$ of the total numbers was found to be phosphate solubilizing isolates. Half of PSA were able to solubilize CIRP was isolated from Shahdad desert of Iran. The mean solubilizing index, concentration of $\mathrm{Pi}$ in solution culture and $\mathrm{pH}$ decrease indicated small and gradual release of $\mathrm{Pi}$ from $\mathrm{CIRP}$ suggesting slow release of $\mathrm{Pi}$ by the isolates. This is an important finding because slow release of $\mathrm{Pi}$ from an insoluble source is one of the characteristics looked for in the preparation of bio-inoculants as biofertilizer. Slow and gradual release could avoid fixation and precipitation of $\mathrm{Pi}$ in many soil types at a wide range of $\mathrm{pH}$. 
Further studies on the biological, chemical and physical soil properties influencing rock phosphate solubilizing actinomycetes at the field would be necessary to evaluate the effectiveness of these potential bio-inoculants for crop improvement. Identification at the molecular level may reveal similarities and dissimilarities between the isolates. The ability of these isolates to also produce other secondary metabolites would give more value added property of the actinomycetes.

\section{References}

1. ACHAL, V., SAVANT, V. \& REDDY, M. S. 2007. Phosphate solubilization by a wild type strain and UV-induced mutants of Aspergillus tubingensis. Soil Biology and Biochemistry, 39, 695-699.

2. AL-HALBOUNI, D., TRABER, J., LYKO, S., WINTGENS, T., MELIN, T., TACKE, D., JANOT, A., DOTT, W. \& HOLLENDER, J. 2008. Correlation of EPS content in activated sludge at different sludge retention times with membrane fouling phenomena. Water Research, 42, 1475-1488.

3. ASUMING-BREMPONG, S. \& AFERI, N. 2014. Isolation of phosphate solubilizing bacteria from tropical soil. Global advanced research journal of agricultural science, 3, 008-015.

4. BAN, J.-G., KIM, H.-B., LEE, M.-J., ANBU, P. \& KIM, E.-S. 2014. Identification of a vitamin D3-specific hydroxylase genes through actinomycetes genome mining. Journal of industrial microbiology and biotechnology, 41, 265-273.

5. BAREA, J., TORO, M., OROZCO, M., CAMPOS, E. \& AZCÓN, R. 2002. The application of isotopic (32P and 15N) dilution techniques to evaluate the interactive effect of phosphate-solubilizing rhizobacteria, mycorrhizal fungi and Rhizobium to improve the agronomic efficiency of rock phosphate for legume crops. Nutrient Cycling in Agroecosystems, 63, 35-42.

6. BRÄNNVALL, E., NILSSON, M., SJÖBLOM, R., SKOGLUND, N. \& KUMPIENE, J. 2014. Effect of residue combinations on plant uptake of nutrients and potentially toxic elements. Journal of environmental management, 132, 287295.

7. BUSARAKAM, K., BULL, A. T., GIRARD, G., LABEDA, D. P., VAN WEZEL, G. P. \& GOODFELLOW, M. 2014. Streptomyces leeuwenhoekii sp. nov., the producer of chaxalactins and chaxamycins, forms a distinct branch in Streptomyces gene trees. Antonie van Leeuwenhoek, 105, 849-861.

8. CASTANHEIRA, N., DOURADO, A. C., ALVES, P. I. L., CORTÉS-PALlERO, A. M., DELGADO-RODRÍGUEZ, A. I., PRAZERES, Â., BORGES, N., SÁNCHEZ, C., CRESPO, M. T. B. \& FARELEIRA, P. 2014. Annual ryegrass-associated bacteria with potential for plant growth promotion. Microbiological Research.

9. COUTINHO, F. P., FELIX, W. P. \& YANO-MELO, A. M. 2012. Solubilization of phosphates in vitro by Aspergillus spp. and Penicillium spp. Ecological Engineering, 42, 85-89.

10. DASTAGER, S. G. \& DAMARE, S. 2013. Marine actinobacteria showing phosphate-solubilizing efficiency in Chorao Island, Goa, India. Current microbiology, 66, 421-427.

11. GANGWAR, M., RANI, S. \& SHARMA, N. 2012. Investigating endophytic actinomycetes diversity from Rice for plant growth promoting and antifungal activity. International Journal of Advanced Life Sciences (IJALS), 1.

12. GUPTA, N., SAHOO, D. \& BASAK, U. C. 2010. Evaluation of in vitro solubilization potential of phosphate solubilizing Streptomyces isolated from phyllosphere of Heritiera fomes (mangrove). African Journal of Microbiology Research, 4, 136-142.

13. HAMDALI, H., BOUIZGARNE, B., HAFIDI, M., LEBRIHI, A., VIROLLE, M. J. \& OUHDOUCH, Y. 2008. Screening for rock phosphate solubilizing Actinomycetes from Moroccan phosphate mines. Applied Soil Ecology, 38, 12-19.

14. KAPRI, A. \& TEWARI, L. 2010. Phosphate solubilization potential and phosphatase activity of rhizospheric Trichoderma spp. Brazilian Journal of Microbiology, 41, 787-795. 
15. KARAGÖZ, K., ATEŞ, F., KARAGÖZ, H., KOTAN, R. \& ÇAKMAKÇı, R. 2012. Characterization of plant growthpromoting traits of bacteria isolated from the rhizosphere of grapevine grown in alkaline and acidic soils. European Journal of Soil Biology, 50, 144-150.

16. KAVIYARASI, K., KANIMOZHI, K., MADHANRAJ, P., PANNEERSELVAM, A. \& AMBIKAPATHY, V. 2011. Isolation, Identification and Molecular Characterization of Phosphate Solubilizing Actinomycetes Isolated From The Coastal Region of Manora, Thanjavur (Dt.). Asian J. Pharm. Tech, 1, 119-122.

17. KHAN, M. S., ZAIDI, A. \& MUSARRAT, J. 2014. Phosphate Solubilizing Microorganisms: Principles and Application of Microphos Technology, Springer.

18. MEHTA, P., WALIA, A., KULSHRESTHA, S., CHAUHAN, A. \& SHIRKOT, C. K. 2014. Efficiency of plant growth promoting $P$ solubilizing Bacillus circulans $C B 7$ for enhancement of tomato growth under net house conditions. Journal of basic microbiology.

19. NESME, T., COLOMB, B., HINSINGER, P. \& WATSON, C. A. 2014. Soil phosphorus management in organic cropping systems: from current practices to avenues for a more efficient use of P resources. Organic farming, prototype for sustainable agricultures. Springer.

20. NGOSONG, C., GABRIEL, E. \& RUESS, L. 2014. Collembola grazing on arbuscular mycorrhiza fungi modulates nutrient allocation in plants. Pedobiologia.

21. OGBO, F. C. 2010. Conversion of cassava wastes for biofertilizer production using phosphate solubilizing fungi. Bioresource Technology, 101, 4120-4124.

22. RAHMAN, M. M., SALLEH, M. A. M., RASHID, U., AHSAN, A., HOSSAIN, M. M. \& RA, C. S. 2014. Production of slow release crystal fertilizer from wastewaters through struvite crystallization. Arabian journal of chemistry, 7, 139155.

23. RAJPUT, A., PANHWAR, Q., NAHER, U., RAJPUT, S., HOSSAIN, E. \& SHAMSHUDDIN, J. 2014. Influence of incubation period, temperature and different phosphate levels on phosphate adsorption in soil. American journal of agricultural and biological sciences, 9, 251.

24. RODRíGUEZ, H. \& FRAGA, R. 1999. Phosphate solubilizing bacteria and their role in plant growth promotion. Biotechnology advances, 17, 319-339.

25. SADEGHI, A., KARIMI, E., DAHAJI, P., JAVID, M., DALVAND, Y. \& ASKARI, H. 2012. Plant growth promoting activity of an auxin and siderophore producing isolate of Streptomyces under saline soil conditions. World Journal of Microbiology and Biotechnology, 28, 1503-1509.

26. SAHU, M. K., SIVAKUMAR, K., THANGARADJOU, T. \& KANNAN, L. 2007. Phosphate solubilizing actinomycetes in the estuarine environment: An inventory. Journal of Environmental Biology, 28, 795-798.

27. SALCEDO, L. D. P., PRIETO, C. \& CORREA, M. F. 2014. Screening phosphate solubilizing actinobacteria isolated from the rhizosphere of wild plants from the eastern Cordillera of the Colombian Andes. African Journal of Microbiology Research, 8, 734-742.

28. SHARMA, P., DAS, R., KALITA, M. C. \& THAKUR, D. 2014. Investigation of extracellular antifungal proteinaceous compound produced by Streptomyces sp. 5K10. African journal of microbiology research, 8, 986-993.

29. STANECK, J. L. \& ROBERTS, G. D. 1974. Simplified approach to identification of aerobic actinomycetes by thinlayer chromatography. Applied microbiology, 28, 226-231.

30. SUJATHA, N. \& AMMANI, K. 2014. Phosphate solubilization by the isolates of fluorescent pseudomonads. International journal of advances in pharmacy, biology and chemistry 3.

31. TAlLAPRAgAdA, P. \& SESHACHALA, U. 2012. Phosphate-solubilizing microbes and their occurrence in the rhizospheres of Piper betel in Karnataka, India. Turk. J. Biol, 36, 25-35.

32. TSAO, H.-W., MICHINAKA, A., YEN, H.-K., GIGLIO, S., HOBSON, P., MONIS, P. \& LIN, T.-F. 2014. Monitoring of geosmin producing Anabaena circinalis using quantitative PCR. Water research, 49, 416-425.

33. VIANI, R. A., RODRIGUES, R. R., DAWSON, T. E., LAMBERS, H. \& OLIVEIRA, R. S. 2014. Soil pH accounts for differences in species distribution and leaf nutrient concentrations of Brazilian woodland savannah and seasonally dry forest species. Perspectives in plant ecology, evolution and systematics, 16, 64-74.

34. WHITELAW, M. 1999. Growth promotion of plants inoculated with phosphate-solubilizing fungi. Advances in Agronomy, 69, 99-151. 